\title{
Unique Size and Shape-Dependent Uptake Behaviors of Non-Spherical Nanoparticles by Endothelial Cells due to a Shearing Flow
}

\author{
Patrick Jurney \\ Rachit Agarwal \\ Vikramjit Singh \\ Krishnendu Roy \\ S.V. Sreenivasan \\ Li Shi, corresponding author
}




\begin{abstract}
The size and shape of nanoparticle (NP) drug carriers can potentially be manipulated to increase the drug delivery efficacy because of their effects on particle margination and interactions with various cells in vivo. It is found in this work that the presence of a physiologically relevant shearing flow rate results in very different size and shape-dependent uptake behavior of negatively charged, non-spherical polyethylene glycol (PEG) hydrogel NPs by endothelial cells (ECs) cultured in a microchannel compared to uptake of either identical NPs in static culture or spherical particles in a shear flow. In particular, larger rod- and disk-shaped PEG NPs show more uptake than smaller ones, opposite to the size effect observed for spherical particles in a flow. Moreover, the trend observed in this dynamic uptake experiment also differs from that reported for uptake of similar PEG NPs by ECs in a static culture, where the intermediate-size disks were found to be uptaken the most. These differences provide experimental support for previous theoretical models, and suggest that the increasing rotational and tumbling motions of larger-size non-spherical NPs in the flow play a dominant role in NP margination and cell interaction, compared to Brownian motion, gravity, and cell membrane deformation energy. These findings point to an important factor in the design and optimization of the size and shape of nanocarriers.
\end{abstract}

\title{
1 INTRODUCTION
}

Nanoparticles (NPs) have shown promise as drug carriers to cancer cells.[1-3] Targeted delivery of nanocarriers specifically to diseased cells can help to shield collateral cells from harmful cytotoxic drugs as well as reduce the many harmful side effects associated with 
traditional chemotherapy. The size, shape, and surface chemistry of NPs can all influence their delivery efficacy to cancerous tissues. NPs, because of their size, cannot enter cells by diffusion alone and rely on active uptake by cells, providing more control over cellular uptake and undesired sequestration by the body.[4] Spherical particles smaller than approximately $10 \mathrm{~nm}$ are rapidly cleared by the kidney glomerulus.[5] In addition, the liver effectively removes particles with characteristic dimension larger than $500 \mathrm{~nm}$ and the spleen filters particles larger than 200 nm.[6,7] NPs with a characteristic dimension less than $500 \mathrm{~nm}$ can be used to exploit the fenestrated endothelium associated with tumor angiogenesis. This phenomenon along with the reduced lymphatic drainage in tumors is known as the enhanced permeation and retention (EPR) effect and has been investigated extensively in order to increase NP delivery efficacy.[8] In addition, endothelial cells (ECs) offer a logical delivery target for many NP-based therapeutics. Spherical NP uptake by ECs has been shown to increase with decreasing size down to approximately $100 \mathrm{~nm}$ for most studies in a static culture, $[9,10]$ and down to $200 \mathrm{~nm}$ under shear stress.[11]

In addition to size, the evolutionarily conserved shapes of viruses and bacteria motivate exploring NP shape as a potentially key factor in enhancing the delivery efficacy.[12-16] Several studies have reported that rod-like particles are more uptaken than their lower aspect ratio, disklike, counterparts and that both tend to be uptaken more and more quickly than volumetrically equivalent spherical particles.[17-20] However some conflicting trends have been reported for gold NPs as well as long worm-like particles of ultra-high aspect ratio where increasing the particle's aspect ratio decreases uptake efficiency.[21-26] In a recent study of the uptake of negatively charged, hydrophilic, polyethylene glycol (PEG) NPs of varying aspect ratios, it was reported that mammalian epithelial and immune cells preferentially internalized disk-shaped 
PEG NPs compared to rod-like and smaller disk-like PEG NPs.[27] In the same study, ECs internalized intermediate-sized disks followed by higher aspect ratio rods, then low aspect ratio rods and larger to smaller disks. ECs also showed significantly higher overall uptake efficiency compared to epithelial cells.[27] These complex and cell line-specific shape/size dependencies were attributed to a competition between three factors: particle-cell membrane adhesion, energy of membrane deformation, and local particle concentration. However, another important factor in NP uptake is the shear stress experienced by ECs, which has been neglected in prior studies of shape-dependent uptake of polymeric rod and disk-like NPs.

Here, we report the shape-dependent uptake of negatively charged, hydrophilic, PEG NPs of varying aspect ratios under physiological shear. Uptake in $100 \mu \mathrm{m}$ high channels was found to be influenced by gravity and differ qualitatively from uptake of identical particles by ECs in static conditions. Larger NPs and NPs with higher aspect ratios show more interaction with, and uptake by, cells because of NP rotation and tumbling in the flow. This factor dominates the other factors that were found to be important in static culture.

\section{MAterial AND Methods}

\subsection{Particle Synthesis}

The four particle shapes used in this study are illustrated in Figure 1. The two largest particles: $800 \times 100 \times 100 \mathrm{~nm}^{3}$ rods and $325 \mathrm{~nm}$ diameter $\times 100 \mathrm{~nm}$ high disks, have volumes within $4 \%$ of each other. The $400 \times 100 \times 100 \mathrm{~nm}^{3}$ rods and $220 \mathrm{~nm}$ diameter $\times 100 \mathrm{~nm}$ high disks have volumes within $5 \%$ of one another. All particles were manufactured using Jet and Flash Imprint Lithography (J-FIL) as described previously.[27,28] 


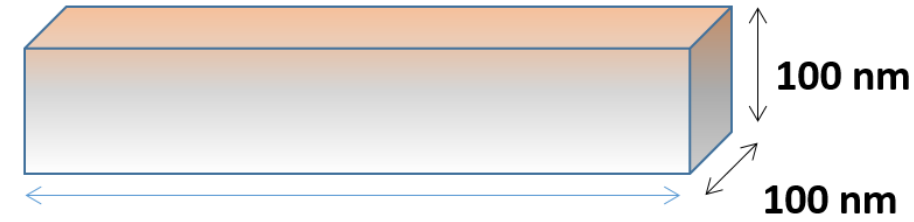

$800 \mathrm{~nm}$

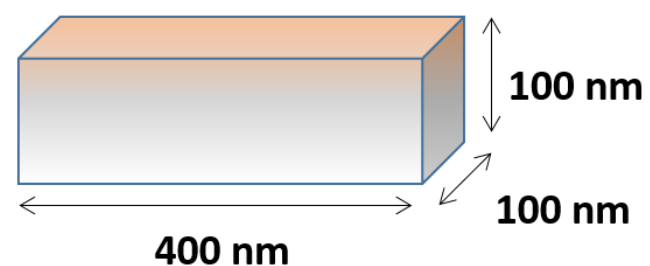

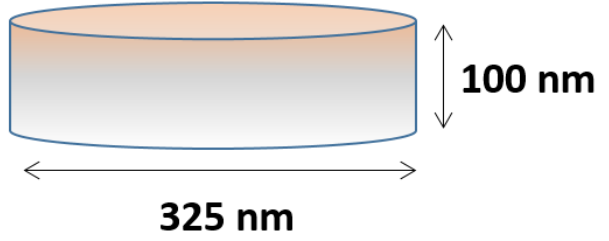

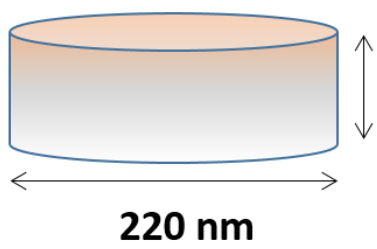

$100 \mathrm{~nm}$

Figure 1: The geometries of the four particle shapes manufactured using the J-FIL process.

\subsection{Cell Culture}

HUVEC (ATCC) cells were cultured in MCDB 131 medium (Life Technologies) supplemented with 10\% fetal bovine serum (Characterized FBS, Hyclone) and 1\% antibiotics (penicillin and streptomycin, Invitrogen) and further supplemented with EGM2 Supplement and growth factor kit (Lonza Inc.). Once confluence was reached in a cell culture flask, cells were removed using $0.25 \%$ trypsin-EDTA (Life Technologies), pelleted and seeded into each of six microchannels ( $\mu$-slide $\mathrm{VI}^{0.1}$, ibidi, LLC) at a density of $5 \times 10^{5}$ cells $/ \mathrm{ml}$. The seeded microchannels were then placed in an incubator at $37{ }^{\circ} \mathrm{C}$ and $5 \% \mathrm{CO}_{2}$ for $1 \mathrm{hr}$ to allow the cells to adhere to the bottom channel wall. Sixty $\mathrm{ml}$ of cell media was then placed in each channel reservoir and replenished at least every 24 hours until cells reached confluence. Once confluence was observed, the channels were connected to a multichannel peristaltic pump with a pulse dampening system[29] as shown in Figure 2. The entire system was then placed in an incubator at $37{ }^{\circ} \mathrm{C}$ and $5 \% \quad \mathrm{CO}_{2}$ and flow of cell media was applied for 24 hours to allow for conformational changes in the cells. 


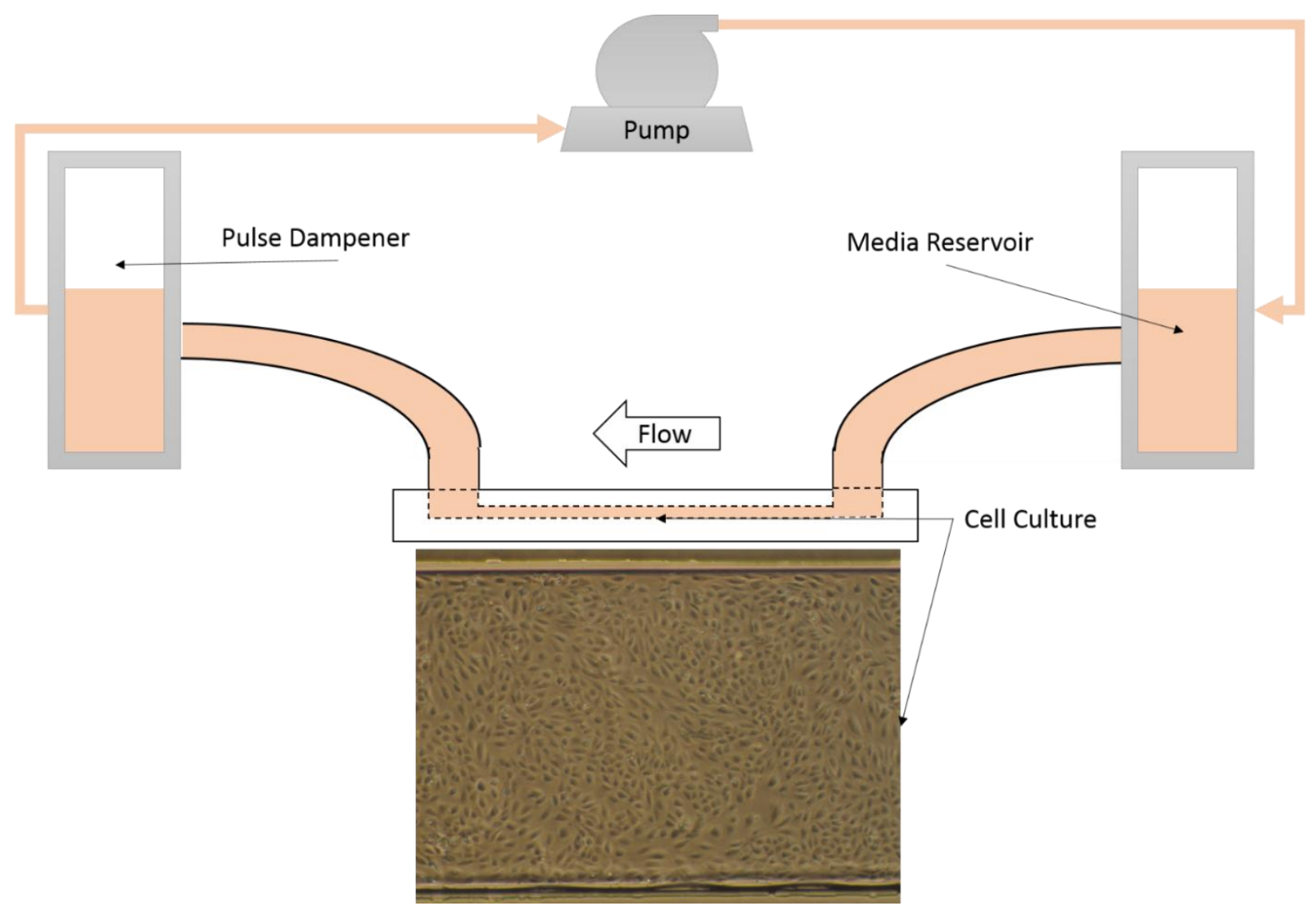

Figure 2: $\quad$ Schematic of the pulse-dampening flow system used to culture HUVECs under shear stress. The micrograph insert is of shear-adapted HUVECs cultured in a $100 \mu \mathrm{m}$ channel using the flow system depicted.

In order to deliver NPs to an inverted culture, ECs were first cultured to confluence identically to the procedure decribed above. However when connecting the peristaltic pump and delivery system, the entire chip containing the six microchannels was inverted 180 degrees and secured such that the ECs were above the flow of media and NPs relative to the dirrection of the gravitational force. 


\subsection{Flow System}

A chip containing six microchannels with a height $(2 h)$ of $100 \mu \mathrm{m}$ and width $(2 w)$ of 1 mm each was connected to a multichannel peristaltic pump with a pulse dampening system as shown in Figure 3. The pump was then turned on at a flow rate of $0.907 \mu 1 / \mathrm{min}$, resulting in a physiologically relevant shear stress of 10 dynes $/ \mathrm{cm}^{2}$ according to the following equations for flow in a finite rectangular duct,

$$
\tau=\eta \frac{Q}{\alpha} \sum_{n=0}^{\infty} \frac{(-1)^{n}}{(2 n+1)^{2}}\left(\frac{2}{\pi}\right) \tanh \left((2 n+1) \frac{\pi h}{2 w}\right)
$$

where

$$
\alpha=\frac{4}{3} h w^{3}-8 w^{4}\left(\frac{2}{\pi}\right)^{5} \sum_{n=0}^{\infty} \frac{1}{(2 n+1)^{5}}\left(\frac{2}{\pi}\right) \tanh \left((2 n+1) \frac{\pi h}{2 w}\right)
$$

and $2 h$ is the channel height, $2 w$ is the channel width, $Q$ is the volumetric flow rate, and $\eta$ is the fluid viscosity.[30] After 24 hours PEG NPs were added to each reservoir at equal fluorescence intensity. Particles were then allowed to flow for 1, 12, or $24 \mathrm{hrs}$. 


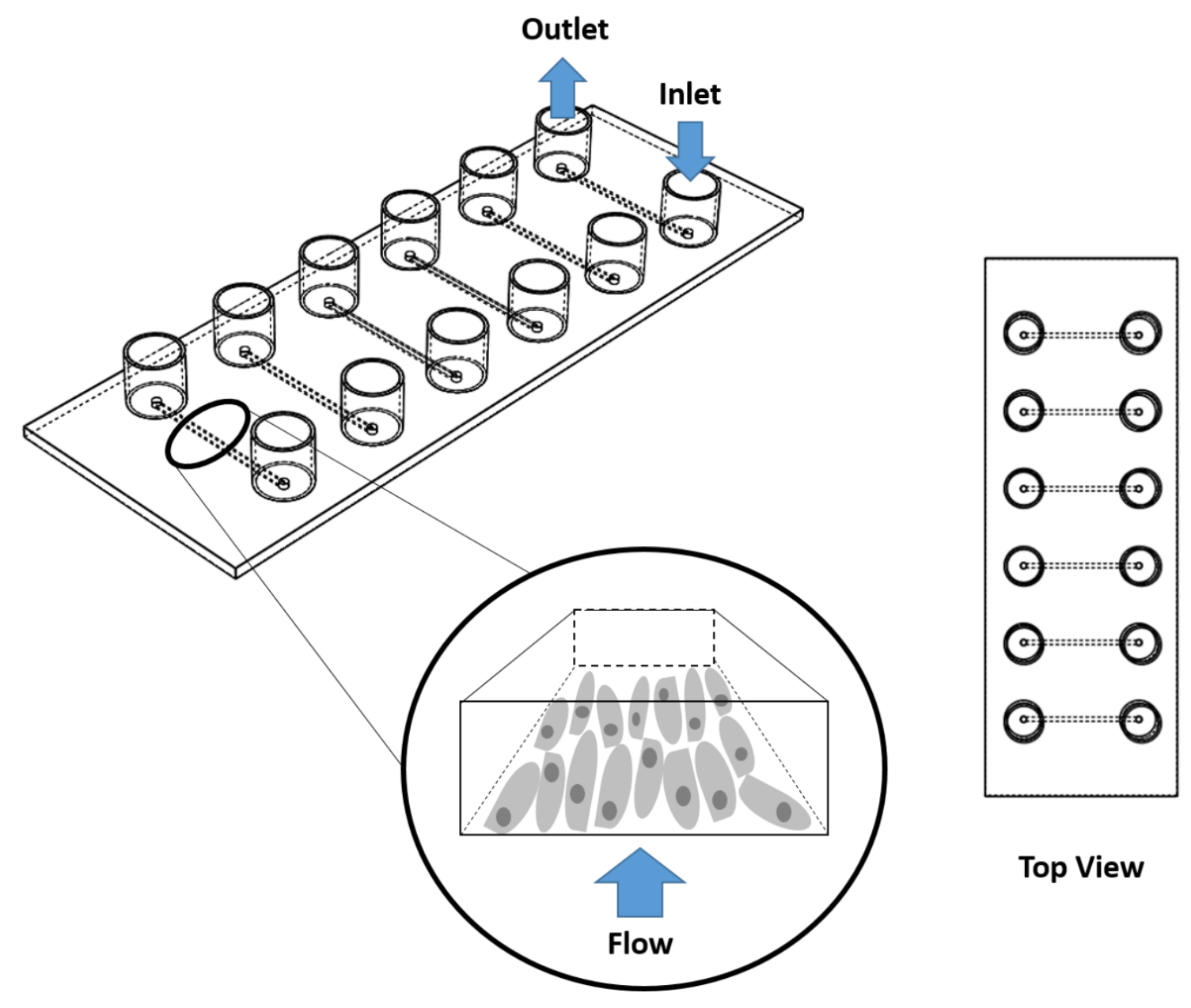

Figure 3: Schematic of the microfluidic chip with six independent channels, $100 \mu \mathrm{m}$ high and $1 \mathrm{~mm}$ wide, with HUVECs cultured on the bottom surface.

\subsection{Cell Staining and Characterization}

Once delivery was complete, the cells were washed once with warm phosphate buffered saline (PBS) and fixed in 4\% paraformaldehyde for 20 min. Excess aldehydes were then quenched by incubating cells with $0.2 \mathrm{M}$ glycine for $10 \mathrm{~min}$. The cells were then permeabilized in $0.1 \%$ Triton $\mathrm{X}-100$ for $10 \mathrm{~min}$. The actin cytoskeleton was labeled by treating the cells for $1 \mathrm{hr}$ in a 1:200 solution of Alexa 594-labeled phalloidin (Invitrogen). Finally the cells were mounted in 
a DAPI-containing mounting medium and imaged using an epifluorescent microscope (Carl Zeiss). Images were taken using three different fluorescent channels showing the actin filaments (Figure 4A), FITC-containing NPs (Figure 4B), and DAPI-stained cell nuclei (Figure 4C). Individual images were then combined into a composite image as shown in Figure 4D.

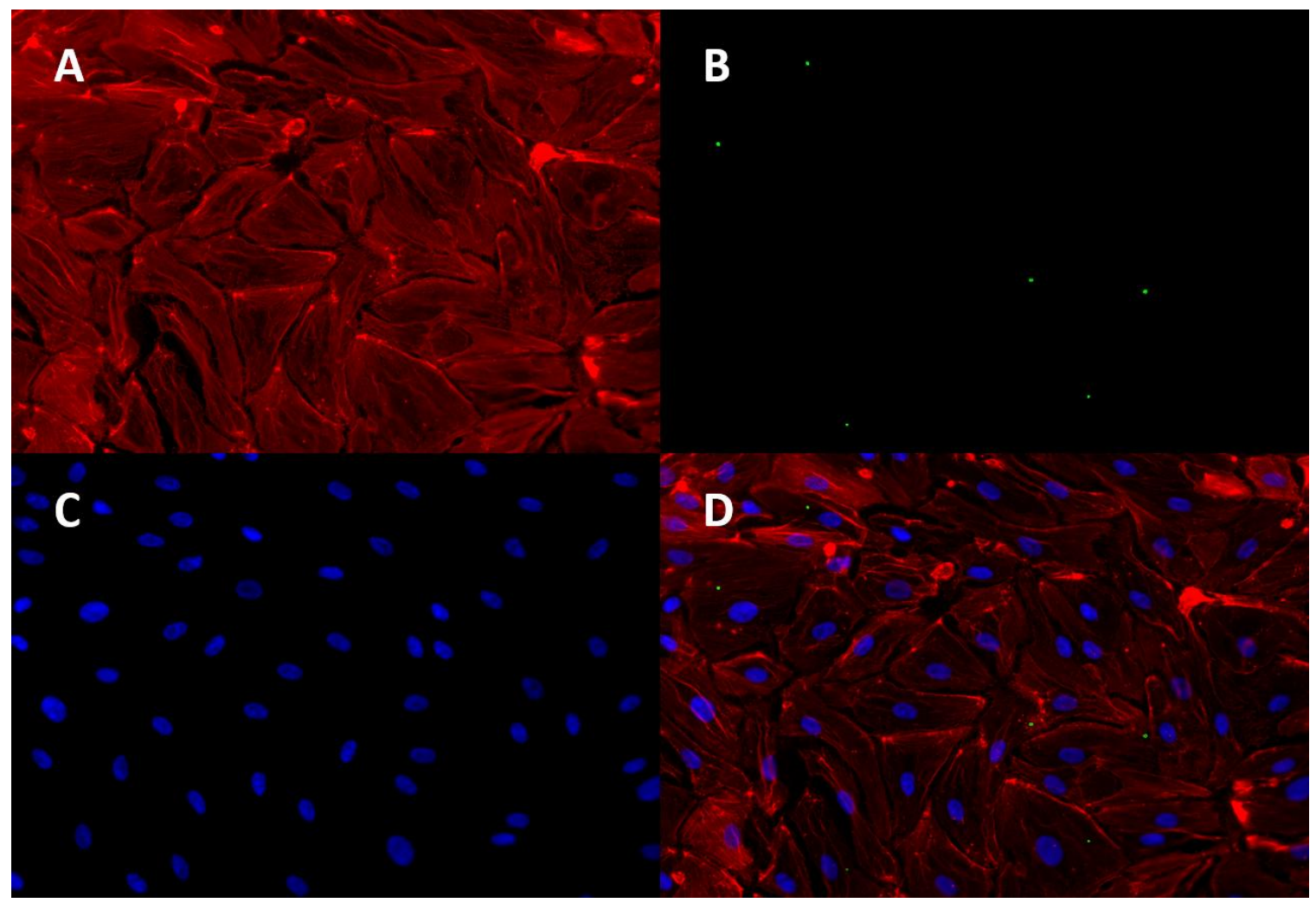

Figure 4: Fluorescence images of: (A) Phalloidin 594-stained actin, (B) FITC-containing PEG nanoparticles, (C) DAPI-stained cell nuclei, and (D) a composite image of all three channels. The images show that cells are confluent and that fluorescent nanoparticles are associated with multiple cells in a single image.

\subsection{Uptake Quantification}

Composite images were used to confirm that fluorescent NPs were co-localized with cells. NPs were then counted along with cell nuclei and each were quantified. The number of particles was then divided by the number of cells (nuclei) for a single image and reported as 
particles/cell. Because the particles/cell for each NP shape was much less than unity, it is statistically unlikely that multiple particles would appear in a single cell. In addition, increasing a NPs charge has been shown to increase stability in several studies.[31,32] Therefore it was assumed that each fluorescent spot was a single particle. Each channel was imaged at six locations, NPs and cells were each quantified, and repeated for each particle type. The error bars represent the random uncertainty with a $95 \%$ confidence interval.

\section{RESULTS}

\subsection{Particle Uptake by Endothelial Cells}

Figure 5A and 5B show the uptake of the four NP shapes delivered to ECs at 1, 12, and 24 hours under shear. Figure 5A shows that $800 \times 100 \times 100 \mathrm{~nm}^{3}$ rods and $325 \mathrm{~nm}$ dimeter $\times 100$ $\mathrm{nm}$ high disks are more uptaken than $400 \times 100 \times 100 \mathrm{~nm}^{3}$ rods and $220 \mathrm{~nm}$ diameter $\times 100 \mathrm{~nm}$ high disks at all three time points. Figure 5B shows that uptake increases over the entire delivery time and that uptake saturation likely does not occur before 24 hours. All brackets indicate a significant statistical difference using a two-tailed t-test with $\mathrm{p}<0.05$. 


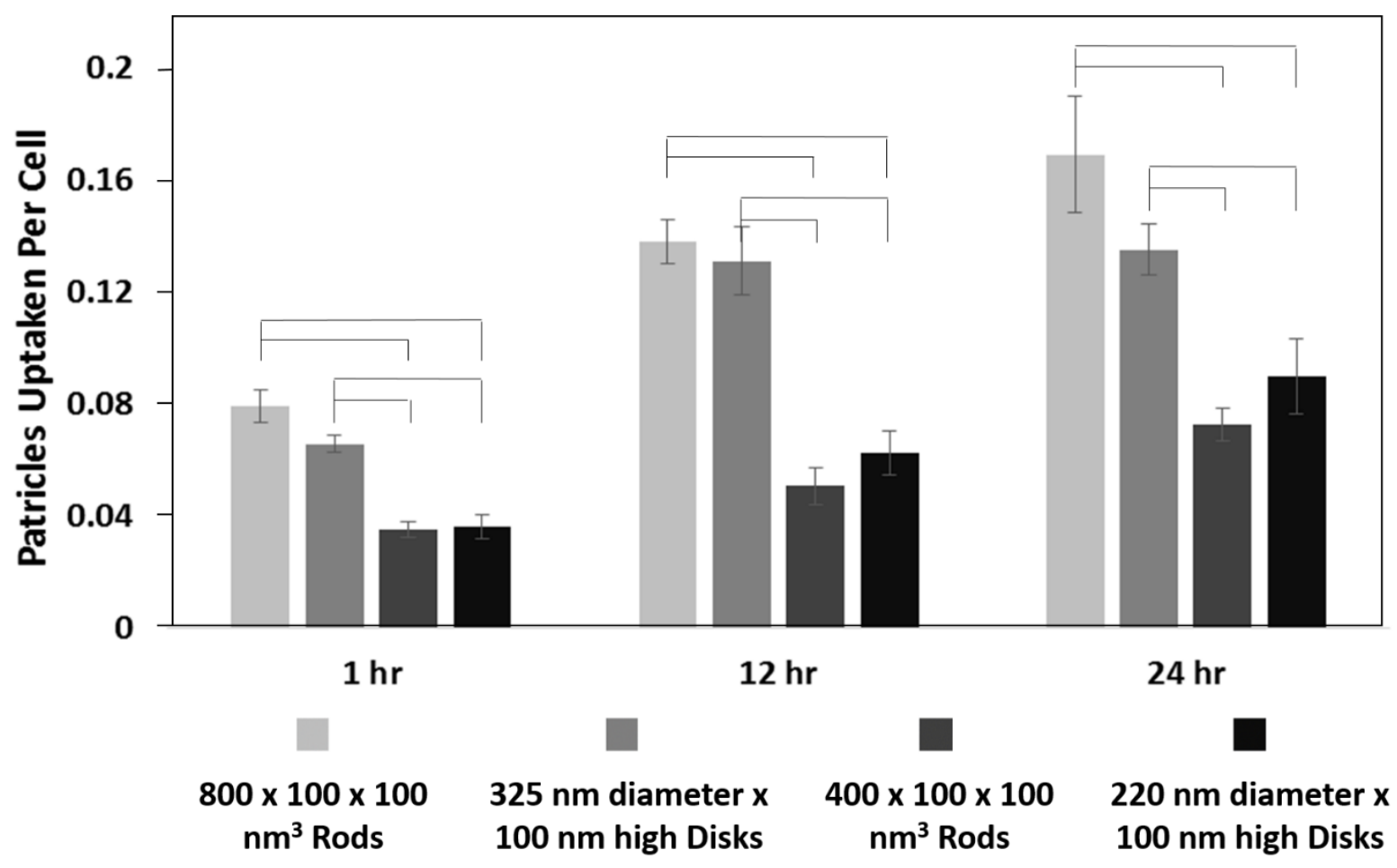

Figure 5: Nanoparticle uptake by human umbilical vein endothelial cells cultured on the bottom of a $100 \mu \mathrm{m}$ channel.

Figure 6 shows the uptake of rod and disk-shaped NPs by ECs cultured on the bottom as well as the top (inverted) of the microfluidic channel after $12 \mathrm{hrs}$. The results show that the 325 $\mathrm{nm}$ diameter $\mathrm{x} 100 \mathrm{~nm}$ high and $220 \mathrm{~nm}$ diameter $\mathrm{x} 100 \mathrm{~nm}$ high disks as well as the $800 \times 100 \mathrm{x}$ $100 \mathrm{~nm}^{3}$ rods are more uptaken by cells cultured on the bottom of the channel than on the top. The $400 \times 100 \times 100 \mathrm{~nm}^{3}$ rods showed no statistical difference in uptake to different surfaces. 


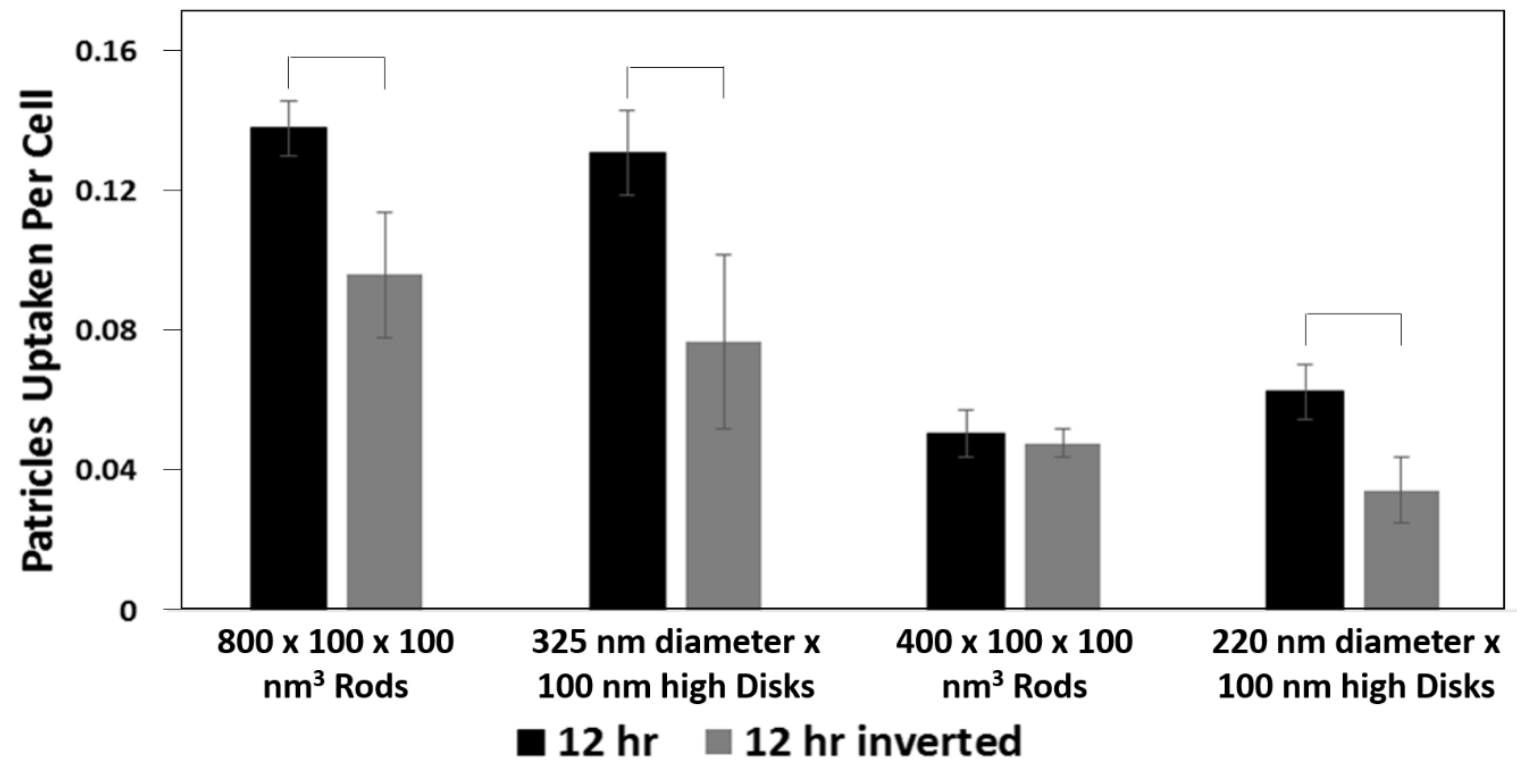

Figure 6: Nanoparticle uptake at twelve hours in channels with cells on the bottom surface (black bars) and with cells on the top surface (grey bars).

In Figure 7 the uptake data by ECs at $24 \mathrm{hr}$ in dynamic culture is compared to that in static culture reported previously.[27] Both data sets were normalized by the maximum uptake at $24 \mathrm{hrs}$ in each study respectively. In static culture $220 \mathrm{~nm}$ diameter x $100 \mathrm{~nm}$ high disks were apparently uptaken more than the $800 \times 100 \times 100 \mathrm{~nm}^{3}$ and $400 \times 100 \times 100 \mathrm{~nm}^{3}$ rods as well as the larger $325 \mathrm{~nm}$ diameter $\times 100 \mathrm{~nm}$ high disks, and smaller $80 \mathrm{~nm}$ diameter $\times 70 \mathrm{~nm}$ high disks[33], which are not shown here. While in dynamic culture $800 \times 100 \times 100 \mathrm{~nm}^{3}$ rods were the most uptaken NP followed by $325 \mathrm{~nm}$ diameter $\mathrm{x} 100 \mathrm{~nm}$ high disks with $220 \mathrm{~nm}$ diameter $\mathrm{x}$ $100 \mathrm{~nm}$ high disks and $400 \times 100 \times 100 \mathrm{~nm}^{3}$ rods the least uptaken NPs. 


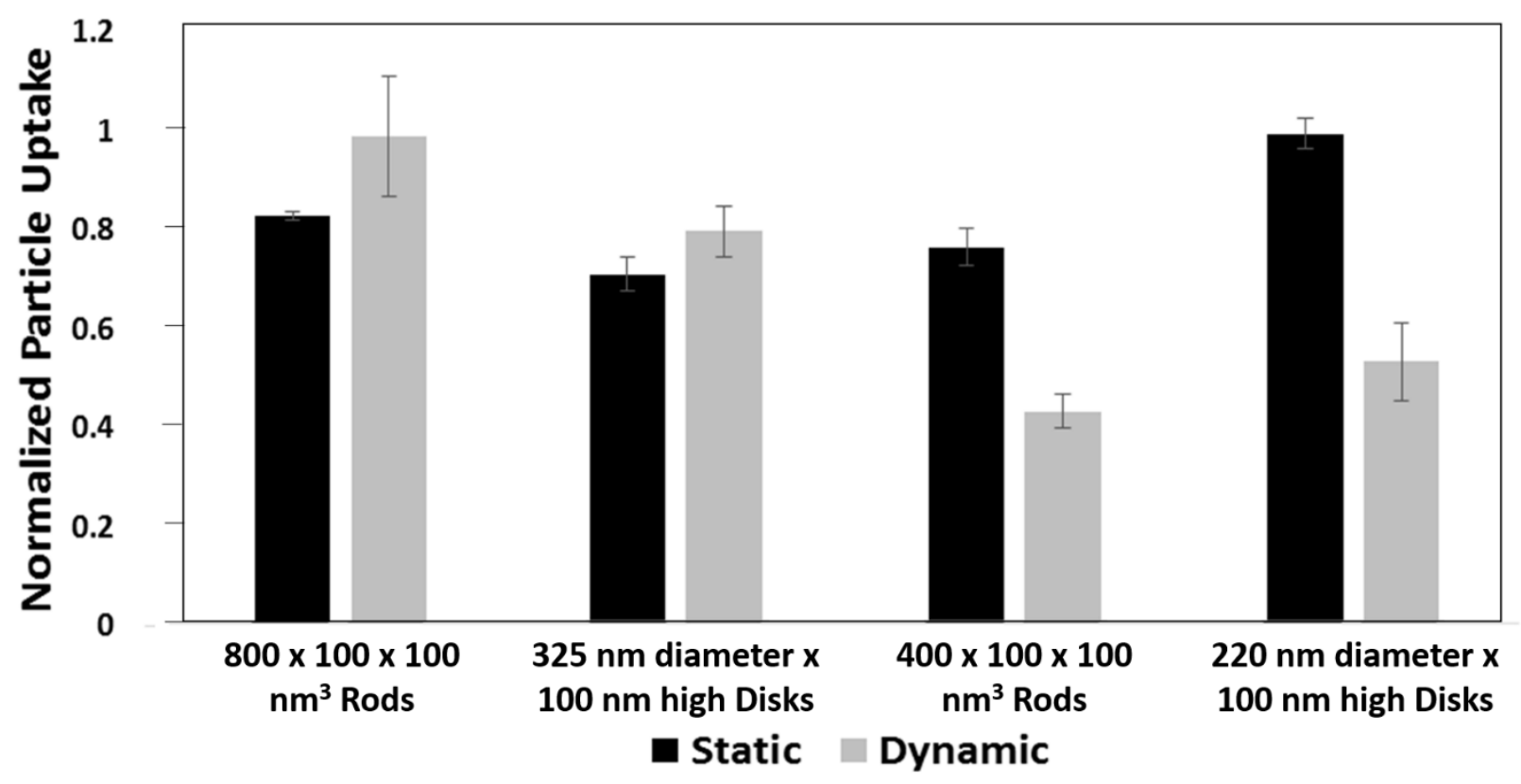

Figure 7: Nanoparticle uptake by endothelial cells in static, NP-containing, media and to the bottom surface of a microchannel under a shear stress of $10 \mathrm{dynes} / \mathrm{cm}^{2}$.

\section{DISCUSSION}

The larger particles $\left(800 \times 100 \times 100 \mathrm{~nm}^{3}\right.$ rods and $325 \mathrm{~nm}$ diameter $\times 100 \mathrm{~nm}$ high disks) being uptaken more than smaller ones $\left(400 \times 100 \times 100 \mathrm{~nm}^{3}\right.$ rods and $220 \mathrm{~nm}$ diameter $\mathrm{x}$ $100 \mathrm{~nm}$ high disks) is contradictory to the trend seen in spherical NP uptake in ECs both in static as well as dynamic uptake studies.[9-11] In those studies uptake increases with decreasing particle diameter. It is worth noting that the smallest dimension of all four NPs used in this study is $100 \mathrm{~nm}$. Gentile et al. reported that spherical particles larger than $500 \mathrm{~nm}$ marginate and adhere to the bottom surface more than smaller ones due to gravity effects[34]. In a complementary modeling study, Lee et al. reported that Brownian diffusive force dominates NP margination rather than gravitational forces for particles smaller than $500 \mathrm{~nm}$ [35]. Both the $800 \mathrm{x}$ $100 \times 100 \mathrm{~nm}^{3}$ rods and $325 \mathrm{~nm}$ diameter $\mathrm{x} 100 \mathrm{~nm}$ high disks used in this study were uptaken 
more by cells on the bottom surface of the microchannel than the top, suggesting a gravity effect on margination and adhesion, and consequently uptake, similar to the behavior reported previously by Gentile et al. for spherical particles with diameter larger than $500 \mathrm{~nm}$.[34] However the $220 \mathrm{~nm}$ diameter $\times 100 \mathrm{~nm}$ high disks showed increased uptake by cells on the bottom of the channel compared to those on the top, while the isovolumetric $400 \times 100 \times 100$ $\mathrm{nm}^{3}$ rods did not. This result suggests that other factors besides just gravity-driven sedimentation affect the difference in the uptake of these shape-specific particles by ECs on the top and bottom surfaces.

Toy et al. have reported that spherical particles smaller than $200 \mathrm{~nm}$ adhere more rapidly to the walls of a microchannel than larger ones, concluding that the Brownian force, which scales as the inverse of the diameter of the particle, was responsible for increased margination of smaller particles.[36] The non-spherical shape of NPs used in this study, again may affect the result of the forces driving margination. For a non-spherical NP, the molecular collisions, which are responsible for Brownian diffusion occurring away from the axes of symmetry of each particle, result partially in translation, similar to spherical particles, but also in rotation. Flowdriven tumbling of non-spherical NPs may also be responsible for the increase in uptake of larger NPs as well as a factor in the differences seen between static and dynamic uptake. As the aspect ratio of a particle increasingly deviates from unity, the eccentric forces cause increased torques and three-dimensional rotation. This increase in aspect ratio and particle tumbling has been associated with increased margination propensity in previous studies.[35-38] Increased eccentricity also decreases the distance between a particle and a cell for two particles whose centers of mass are the same distance from a cell, effectively increasing the uptake probability. Therefore the increased flow-driven tumbling of larger and larger-aspect ratio particles increases 
the frequency of interaction between particles and cells. This property of non-spherical particles, dominates Brownian diffusion of smaller particles, the driving factor in small spherical particles being uptaken more in static and dynamic EC cultures.

\section{Conclusions}

The experiment has obtained unique size and shape- dependent uptake behaviors of negatively charged, hydrophilic, PEG NPs due to the presence of a physiologically relevant shear stress. The results show that that the larger $800 \times 100 \times 100 \mathrm{~nm}^{3}$ rods and $325 \mathrm{~nm}$ diameter $\times 100$ $\mathrm{nm}$ high disks are more uptaken than the smaller $400 \times 100 \times 100 \mathrm{~nm}^{3}$ rods and $220 \mathrm{~nm}$ diameter x $100 \mathrm{~nm}$ high disks at 1, 12, and 24 hours. The experiment has also shown that the gravity force results in less uptake of the $800 \times 100 \times 100 \mathrm{~nm}^{3}$ rods as well as the $325 \mathrm{~nm}$ diameter $\times 100 \mathrm{~nm}$ high and $220 \mathrm{~nm}$ diameter $\mathrm{x} 100 \mathrm{~nm}$ high disks are uptaken less by cells cultured on the top surface than those on the bottom surface of the microchannel. The trend of larger particles being uptaken more than smaller ones observed here is opposite to that of similarly sized spherical particles reported in the literature. The size and aspect ratio dependencies observed for the particles in the flow are also different from the cell-type specific size and shape dependence observed in static culture. The difference reveals that the increased rotation of larger and larger aspect ratio nanoparticles in the flow increases the particle-cell interaction, and this factor dominates other competing effects such as decreased Brownian diffusion and increased membrane wrapping energy with increasing size. The observation of increased cell uptake of larger size or aspect ratio particles in a flow and the underlying mechanisms can inform the rational design of NC size and shape to optimize drug delivery efficacy. 


\section{ACKNOWLEDGEMENTS}

This work is supported by National Science Foundation (NSF) Award No. CMMI-0900715.

The authors graciously acknowledge technical and material support from Professor Jeanne Stachowiak and Professor Aaron Baker.

\section{REFERENCES}

[1] L. Brannon-Peppas, J.O. Blanchette, Nanoparticle and targeted systems for cancer therapy, Adv. Drug Deliv. Rev. 64 (2012) 206-212. doi:10.1016/j.addr.2012.09.033.

[2] I. Brigger, C. Dubernet, P. Couvreur, Nanoparticles in cancer therapy and diagnosis, Adv. Drug Deliv. Rev. 64 (2012) 24-36. doi:10.1016/j.addr.2012.09.006.

[3] A.Z. Wang, R. Langer, O.C. Farokhzad, Nanoparticle Delivery of Cancer Drugs, Annu. Rev. Med. 63 (2012) 185-198. doi:10.1146/annurev-med-040210-162544.

[4] D. Smith, P. Artursson, A. Avdeef, L. Di, G.F. Ecker, B. Faller, J.B. Houston, M. Kansy, E.H. Kerns, S.D. Krämer, H. Lennernäs, H. van de Waterbeemd, K. Sugano, B. Testa, Passive lipoidal diffusion and carrier-mediated cell uptake are both important mechanisms of membrane permeation in drug disposition., Mol. Pharm. 11 (2014) 1727-38. doi:10.1021/mp400713v.

[5] H.S. Choi, W. Liu, P. Misra, E. Tanaka, J.P. Zimmer, B. Itty Ipe, M.G. Bawendi, J. V Frangioni, Renal clearance of quantum dots., Nat. Biotechnol. 25 (2007) 1165-1170. doi:10.1038/nbt1340.

[6] P. Decuzzi, B. Godin, T. Tanaka, S.-Y. Lee, C. Chiappini, X. Liu, M. Ferrari, Size and shape effects in the biodistribution of intravascularly injected particles., J. Control. Release. 141 (2010) 320-7. doi:10.1016/j.jconrel.2009.10.014.

[7] R. Agarwal, K. Roy, Intracellular delivery of polymeric nanocarriers: a matter of size, shape, charge, elasticity and surface composition., Ther. Deliv. 4 (2013) 705-23. doi:10.4155/tde.13.37.

[8] F. Alexis, E. Pridgen, L.K. Molnar, O.C. Farokhzad, Factors affecting the clearance and biodistribution of polymeric nanoparticles., Mol. Pharm. 5 505-15. doi:10.1021/mp800051m.

[9] A. Lin, A. Sabnis, S. Kona, S. Nattama, H. Patel, J.-F. Dong, K.T. Nguyen, Shearregulated uptake of nanoparticles by endothelial cells and development of endothelialtargeting nanoparticles., J. Biomed. Mater. Res. A. 93 (2010) 833-42. doi:10.1002/jbm.a.32592.

[10] C. He, Y. Hu, L. Yin, C. Tang, C. Yin, Effects of particle size and surface charge on cellular uptake and biodistribution of polymeric nanoparticles., Biomaterials. 31 (2010) 3657-66. doi:10.1016/j.biomaterials.2010.01.065.

[11] S. Kona, J.-F. Dong, Y. Liu, J. Tan, K.T. Nguyen, Biodegradable nanoparticles mimicking 
platelet binding as a targeted and controlled drug delivery system., Int. J. Pharm. 423 (2012) 516-24. doi:10.1016/j.ijpharm.2011.11.043.

[12] A.A. Brakhage, S. Bruns, A. Thywissen, P.F. Zipfel, J. Behnsen, Interaction of phagocytes with filamentous fungi., Curr. Opin. Microbiol. 13 (2010) 409-15.

doi:10.1016/j.mib.2010.04.009.

[13] S.S. Justice, D.A. Hunstad, L. Cegelski, S.J. Hultgren, Morphological plasticity as a bacterial survival strategy., Nat. Rev. Microbiol. 6 (2008) 162-8. doi:10.1038/nrmicro1820.

[14] E.A. Simone, T.D. Dziubla, V.R. Muzykantov, Polymeric carriers: role of geometry in drug delivery., Expert Opin. Drug Deliv. 5 (2008) 1283-300. doi:10.1517/17425240802567846.

[15] J. a Champion, Y.K. Katare, S. Mitragotri, Particle shape: a new design parameter for micro- and nanoscale drug delivery carriers., J. Control. Release. 121 (2007) 3-9. doi:10.1016/j.jconrel.2007.03.022.

[16] S. Venkataraman, J.L. Hedrick, Z.Y. Ong, C. Yang, P.L.R. Ee, P.T. Hammond, Y.Y. Yang, The effects of polymeric nanostructure shape on drug delivery., Adv. Drug Deliv. Rev. 63 (2011) 1228-46. doi:10.1016/j.addr.2011.06.016.

[17] S. Barua, J.-W. Yoo, P. Kolhar, A. Wakankar, Y.R. Gokarn, S. Mitragotri, Particle shape enhances specificity of antibody-displaying nanoparticles., Proc. Natl. Acad. Sci. U. S. A. 110 (2013) 3270-5. doi:10.1073/pnas.1216893110.

[18] N. Hao, L. Li, Q. Zhang, X. Huang, X. Meng, Y. Zhang, D. Chen, F. Tang, L. Li, The shape effect of PEGylated mesoporous silica nanoparticles on cellular uptake pathway in Hela cells, Microporous Mesoporous Mater. 162 (2012) 14-23.

doi:10.1016/j.micromeso.2012.05.040.

[19] S.E.A. Gratton, P.A. Ropp, P.D. Pohlhaus, J.C. Luft, V.J. Madden, M.E. Napier, J.M. DeSimone, The effect of particle design on cellular internalization pathways, Proc. Natl. Acad. Sci. 105 (2008) 11613-11618. doi:10.1073/pnas.0801763105.

[20] Y. Qiu, Y. Liu, L. Wang, L. Xu, R. Bai, Y. Ji, X. Wu, Y. Zhao, Y. Li, C. Chen, Surface chemistry and aspect ratio mediated cellular uptake of Au nanorods., Biomaterials. 31 (2010) 7606-19. doi:10.1016/j.biomaterials.2010.06.051.

[21] J.A. Champion, S. Mitragotri, Shape Induced Inhibition of Phagocytosis of Polymer Particles, Pharm. Res. 26 (2008) 244-249. doi:10.1007/s11095-008-9626-Z.

[22] K. Zhang, H. Fang, Z. Chen, J.-S.A. Taylor, K.L. Wooley, Shape effects of nanoparticles conjugated with cell-penetrating peptides (HIV Tat PTD) on CHO cell uptake., Bioconjug. Chem. 19 (2008) 1880-7. doi:10.1021/bc800160b.

[23] L. Florez, C. Herrmann, J.M. Cramer, C.P. Hauser, K. Koynov, K. Landfester, D. Crespy, V. Mailänder, How shape influences uptake: interactions of anisotropic polymer nanoparticles and human mesenchymal stem cells., Small. 8 (2012) 2222-30. doi:10.1002/smll.201102002.

[24] Y. Zhang, S. Tekobo, Y. Tu, Q. Zhou, X. Jin, S.A. Dergunov, E. Pinkhassik, B. Yan, Permission to enter cell by shape: nanodisk vs nanosphere., ACS Appl. Mater. Interfaces. 4 (2012) 4099-105. doi:10.1021/am300840p. 
[25] O. Shimoni, Y. Yan, Y. Wang, F. Caruso, Shape-dependent cellular processing of polyelectrolyte capsules., ACS Nano. 7 (2013) 522-30. doi:10.1021/nn3046117.

[26] J.-W. Yoo, N. Doshi, S. Mitragotri, Endocytosis and Intracellular Distribution of PLGA Particles in Endothelial Cells: Effect of Particle Geometry., Macromol. Rapid Commun. 31 (2010) 142-8. doi:10.1002/marc.200900592.

[27] R. Agarwal, V. Singh, P. Jurney, L. Shi, S. V Sreenivasan, K. Roy, Mammalian cells preferentially internalize hydrogel nanodiscs over nanorods and use shape-specific uptake mechanisms., Proc. Natl. Acad. Sci. U. S. A. 110 (2013) 17247-52. http://www.ncbi.nlm.nih.gov/pubmed/24101456.

[28] R. Agarwal, V. Singh, P. Jurney, L. Shi, S. V. Sreenivasan, K. Roy, Scalable imprinting of shape-specific polymeric nanocarriers using a release layer of switchable water solubility, ACS Nano. 6 (2012) 2524-2531.

[29] P.L. Voyvodic, D. Min, A.B. Baker, A multichannel dampened flow system for studies on shear stress-mediated mechanotransduction., Lab Chip. 12 (2012) 3322-30. doi:10.1039/c2lc40526a.

[30] R.J. Cornish, Flow in a Pipe of Rectangular Cross-Section, Proc. R. Soc. A Math. Phys. Eng. Sci. 120 (1928) 691-700. doi:10.1098/rspa.1928.0175.

[31] V. Ayala, A.P. Herrera, M. Latorre-Esteves, M. Torres-Lugo, C. Rinaldi, Effect of surface charge on the colloidal stability and in vitro uptake of carboxymethyl dextran-coated iron oxide nanoparticles., J. Nanopart. Res. 15 (2013) 1874. doi:10.1007/s11051-013-1874-0.

[32] R.A. French, A.R. Jacobson, B. Kim, S.L. Isley, R.L. Penn, P.C. Baveye, Influence of Ionic Strength, $\mathrm{pH}$, and Cation Valence on Aggregation Kinetics of Titanium Dioxide Nanoparticles, Environ. Sci. Technol. 43 (2009) 1354-1359. doi:10.1021/es802628n.

[33] R. Agarwal, V. Singh, P. Jurney, L. Shi, S. V Sreenivasan, K. Roy, Mammalian cells preferentially internalize hydrogel nanodiscs over nanorods and use shape-specific uptake mechanisms., Proc. Natl. Acad. Sci. U. S. A. 110 (2013) 17247-52. doi:10.1073/pnas.1305000110.

[34] F. Gentile, A. Curcio, C. Indolfi, M. Ferrari, P. Decuzzi, The margination propensity of spherical particles for vascular targeting in the microcirculation., J. Nanobiotechnology. 6 (2008) 9. doi:10.1186/1477-3155-6-9.

[35] S.-Y. Lee, M. Ferrari, P. Decuzzi, Shaping nano-/micro-particles for enhanced vascular interaction in laminar flows., Nanotechnology. 20 (2009) 495101. doi:10.1088/09574484/20/49/495101.

[36] R. Toy, E. Hayden, C. Shoup, H. Baskaran, E. Karathanasis, The effects of particle size, density and shape on margination of nanoparticles in microcirculation., Nanotechnology. 22 (2011) 115101.

http://www.pubmedcentral.nih.gov/articlerender.fcgi?artid=3530262\&tool=pmcentrez\&re ndertype $=$ abstract (accessed February 20, 2015).

[37] A.J. Thompson, E.M. Mastria, O. Eniola-Adefeso, The margination propensity of ellipsoidal micro/nanoparticles to the endothelium in human blood flow, Biomaterials. 34 (2013) 5863-5871. doi:10.1016/j.biomaterials.2013.04.011.

[38] P. Decuzzi, R. Pasqualini, W. Arap, M. Ferrari, Intravascular delivery of particulate 
systems: does geometry really matter?, Pharm. Res. 26 (2009) 235-43. doi:10.1007/s11095-008-9697-x. 

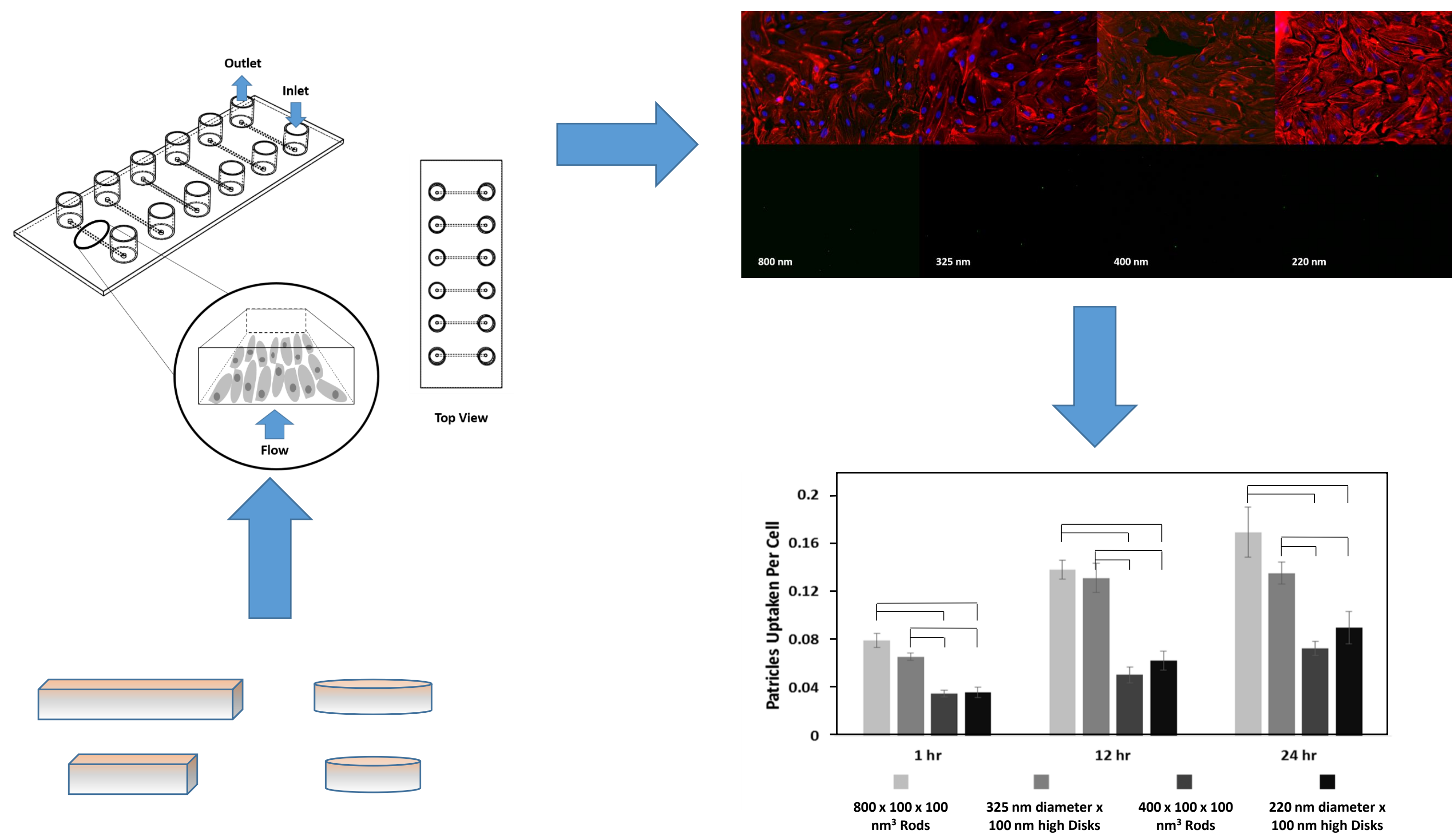